\title{
Asteroid astrometry as a link between ICRF and the Dynamical Reference Frames
}

\author{
D. A. Nedelcu ${ }^{1}$, J. Souchay ${ }^{2}$, M. Birlan ${ }^{3}$, P. P. Popescu ${ }^{1}$, \\ P. V. Paraschiv ${ }^{1}$ and O. Badescu ${ }^{1}$ \\ ${ }^{1}$ Astronomical Institute of the Romanian Academy, \\ Cutitul de Argint 5, RO-040557, Bucharest, Romania \\ email: [nedelcu, petre, paras, octavian] @aira.astro.ro \\ ${ }^{2}$ SYRTE Observatoire de Paris, \\ 61, avenue de l'Observatoire 75014, Paris, France \\ email: jean. souchay@obspm.fr \\ ${ }^{3}$ IMCCE Observatoire de Paris, \\ 61, avenue de l'Observatoire 75014, Paris, France \\ email: mirel.birlan@obspm.fr
}

\begin{abstract}
Highly accurate astrometry of asteroids in the frame of QSOs will provide the direct link between the Dynamical Reference Frame and the International Celestial Reference Frame. We propose a procedure that implies a selection of events for asteroids with accurately determined orbits crossing the CCD field containing the selected quasars. For asteroid ephemerides, a numerical integration method coupled with precise modelling of asteroid brightness will be used for analyzing our observations. A list of predictions for this type of "close approaches" will be presented.
\end{abstract}

Keywords. astrometry, minor planets, asteroids, reference systems

\section{Introduction}

In this paper we propose a project on the link between Dynamical Reference Frame (DRF) and the International Celestial Reference Frame (ICRF) by means of close angular approaches of asteroids and quasars. ICRF is the radio realization of International Celestial Reference System and is formed by VLBI radio positions of 212 extragalactic radiosources (quasars, galaxies, BL Lac objects) distributed over the entire sky and selected by astrometrical stability criteria. Realization of ICRS at optical wavelengths is given by Hipparcos Celestial Reference Frame (HCRF). The DRF is a realization of a dynamical reference system and it is defined by the ephemerides of one or more solar system bodies. The curent ICRF with 717 radiosources has an average sky density of $\approx$ one source per $9^{\circ} \times 9^{\circ}$. This makes difficult direct observations of a quasar and an asteroid in the same field of view (FOV). Thus, in order to investigate the link between the $\mathrm{DRF}$ and ICRF we need a much larger sample of extragalactic objects directly accesible to observations. The obvious choice is the Vèron-Cetty \& Vèron list of 85221 quasars (Fig. 1).

\section{Methods}

Accurate positions for quasars could be obtained : 1) from USNO B1.0 plates using UCAC2 and 2MASS as astrometric reference catalogs (Andrei et al. 2004) or 2) by direct astrometry of quasars in the optical domain (Assafin et al. 2007). While the first method is fully automated the second one allow us to directly obtain astrometric positions for 


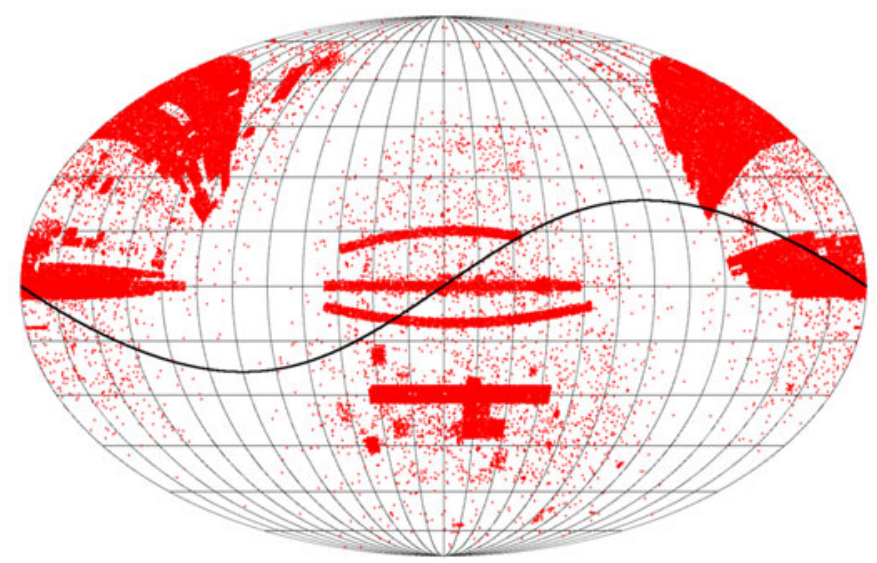

Figure 1. All sky distribution of Veron-Cetty \& Veron 85221 quasars. Image center has $\alpha=0 h, \delta=0^{\circ}$ coordinates. Black line represents the ecliptic.

quasars and the nearby asteroids using the same set of reference stars. In 2004 at the Belogradchick Observatory $\left(\lambda=-22^{\circ} 40^{\prime} 30^{\prime \prime} ; \phi=+43^{\circ} 37^{\prime} 22^{\prime \prime} ; \mathrm{h}=650 \mathrm{~m}\right)$, Bulgaria we started an observational program aiming at densifying the northern hemisphere coverage with precise astrometric ICRF source positions. Observations were carried out using a 0.60m Zeiss telescope (f/12.5) equipped with an Apogee 47P CCD detector of $1024 \times 1024$ pixels of $13 \mu \mathrm{m}$ size. The detector was operated in a $2 \times 2$ binned mode resulting on a FoV of $6.16^{\prime} \times 6.16^{\prime}$ with an image scale of 0 ". 72 / pixel. From a set of 59 northern ICRF sources (their optical counterparts) we obtained the average and standard deviation for the optical minus radio position offsets of +6 mas (51mas) and +7 mas (57mas). The optical CCD source positions were referred to the UCAC2 catalog - the best HCRF representative available to date in terms of stellar density, position and proper motion accuracy. Asteroid positions are obtained in the framework of an accurate, fully relativistic solar system integration for the 8 planets, Pluto and the largest 3 asteroids (Ceres, Pallas, Vesta). The integrator uses a 12-order Bulirsch-Stoer method of Bader and Deuflhard with automatic step size control provided by Gnu Scientific Library (GSL). The initial conditions for the asteroid are obtained from the latest osculating elements (Bowell et al. 1994). Beside selecting asteroids with a good orbit-quality parameter (OQP), we also consider the effect of photocenter displacement (for the minor bodies of known sizes) by numerically computing the surface luminosity distribution, corresponding to asteroids obeying two different scattering laws (Lommel-Seeliger and Minnaert). Finally we run a search for all the forthcoming close approaches between selected asteroids and quasars in 2008. For these events to be observable by our instrumentation we impose two conditions: a maximum magnitude of 18.0 for the quasar and a maximum angular separation for the two objects of $10^{\prime}$. The complete list of events is available at http://observer.astro.ro/QA.

\section{References}

Andrei, A. H., Fienga, A., Assafin, M., Penna, J. L., da Silva Neto, D. N., \& Vieira Martins, R. 2004, Journées 2004 - systèmes de reférénce spatio-temporels., p. 228

Assafin, M., Nedelcu, D. A., Badescu, O., Popescu, P., Andrei, A. H., Camargo, J. I. B., da Silva Neto, D. N., \& Vieira Martins R. 2007, $A \mathscr{E} A$, in press

Bowell, E., Muinonen, K., \& Wasserman, L. H. 1994, Asteroids, comets, meteors 1993, p. 477

Vèron-Cetty M.-P. \& Vèron P. 2006, A\&A A, 455, 733 